\title{
Desarrollo de competencias transversales mediante tutoría virtual
}

\section{José Manuel Navarro Jover ${ }^{\mathrm{a}}$ y Françoise Olmo Cazevieille ${ }^{\mathrm{b}}$}

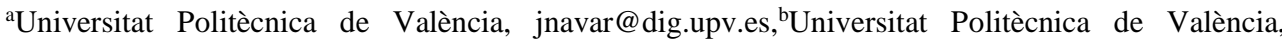
folmo@idm.upv.es, GIIMA (Grupo de Investigación e Innovación en Metodologías Activas)

\begin{abstract}
The aim of this study, in the context of the Polytechnic University of Valencia, is to develop and assess two generic competences: comprehension and assimilation, as well as teamwork and leadership, through virtual tutorials with a web 2.0 tool.

Once specific tasks have been designed for two different subjects, the online Vyew.com application was used for monitoring and assessment through virtual tutorials carried out between students and teacher groups.

In order to assess the degree of acquisition of these competences, a special rubric was developed. Moreover, a final survey of students' opinions was conducted with the aim of finding out about how they view their own progress in these competences and to make them aware of the importance of training in their acquisition.

Keywords: Virtual tutorials, generic competences, teamwork, understanding and integration.
\end{abstract}

\section{Resumen}

Este trabajo pretende, dentro del marco de la formación en competencias transversales de la Universitat Politècnica de València, desarrollar y evaluar la competencia de comprensión e integración y la competencia de trabajo en equipo y liderazgo, mediante tutorías virtuales con una herramienta web 2.0.

Una vez diseñadas unas tareas específicas para dos asignaturas distintas, se ha utilizado la aplicación online Vyew.com para su seguimiento y evaluación mediante sesiones de tutorías virtuales conjuntas entre grupos de alumnos y profesor.

La evaluación del grado de adquisición de dichas competencias se ha llevado a cabo mediante una rúbrica elaborada y adaptada a la actividad propuesta. Paralelamente se realizó una encuesta final a los alumnos para conocer su 
opinión acerca de su progreso en estas competencias, así como para que tomen conciencia de la importancia de la formación en las mismas.

Palabras clave: tutoría virtual, competencia transversal, trabajo en equipo, comprensión e integración.

\section{Introducción}

La creación del Espacio Europeo de Educación Superior (EEES) iniciado con el Tratado de Bolonia (1999) ha llevado consigo la remodelación de las titulaciones con el fin de armonizar la estructura de los estudios entre los países miembros, favorecer la movilidad y ofrecer más empleo. En este contexto, nació el proyecto Tuning (González y Wagenaar, 2006) para establecer puntos de referencia para el diseño y la ejecución de programas de titulaciones. En su fase 1 (2000-2002) ya se ponía de manifiesto que "debería prestarse atención y tiempo al desarrollo de lo que se conoce como competencias genéricas o habilidades transferibles" (González y Wagenaar, 2006: 12). Y, se llegó a la conclusión de que la mejor manera de conseguir los mejores resultados sería integrar actividades de aprendizaje y enseñanza dirigidas a la formación en competencias genéricas dentro de un proceso vinculado con las competencias específicas de cada área (ibídem). En este sentido, la UPV ha señalado trece competencias transversales clave para que sus estudiantes puedan adaptarse a cualquier cambio en sus situaciones profesionales a lo largo de sus vidas. La verificación y evaluación de dichas competencias se pondrán en marcha el próximo curso. Esto implica unas modificaciones en la planificación docente ya que, a nuestro entender, a los tres pilares básicos definidos por Harris y Hofer (2009) como el conocimiento disciplinar, pedagógico y tecnológico, se debe añadir el conocimiento en competencias genéricas.

En este sentido, este trabajo se centra en contribuir en el desarrollo de dos de ellas: la comprensión y la integración (competencia 1) por una parte y el trabajo en equipo y liderazgo (competencia 6) por otra, mediante la utilización de una herramienta 2.0 (DiNucci, 1999), el Vyew.com. Partiendo de un estudio anterior (Olmo y Navarro, 2014) sobre la tutoría virtual en la enseñanza universitaria en el que se investigaba por un lado, los beneficios de ésta en relación con la presencial en cuanto a solventar las incompatibilidades horarias o la imposibilidad de desplazarse por trabajo u otros motivos y por otro, el medio de ayudar al alumnado a llevar a bien la realización de sus trabajos, se plantean aquí unas actividades diseñadas para no solamente desarrollar las competencias que acabamos de mencionar sino también recoger evidencias de su adquisición. 


\section{Objetivos}

El objetivo general de este trabajo es desarrollar las competencias transversales 1 (comprensión e integración) y 6 (trabajo en equipo y liderazgo) mediante tutorías virtuales con la herramienta Vyew.com e implementar estrategias o procedimientos para la recogida de evidencias de cara a la evaluación de la adquisición de dichas competencias.

Con la adquisición de la competencia 1, se pretende que los alumnos alcancen un grado de reflexión que les permita explicar y relacionar los conceptos clave de la asignatura; con la competencia 6, que trabajen en equipo de una manera cooperativa, implicada, responsable y eficaz. Cabe señalar que para esta competencia 6 , no se ha considerado el aspecto del liderazgo, por ser los grupos de trabajo de tamaño reducido.

Se parte de las siguientes hipótesis:

- La tutoría virtual ofrece una mayor flexibilidad en lo referente a la gestión y adaptación del tiempo, y sincronización entre los alumnos entre sí y con el profesor, puesto que permite trabajar desde casa.

- La tutoría virtual complementa la clase presencial ya que permite aclarar dudas, orientar y explicar de forma más personalizada.

- La tutoría virtual permite recoger evidencias de la adquisición de las competencias señaladas porque al conservar los trabajos de los alumnos estos siempre están disponibles para ellos y para el profesor. Además, se evita el tener que imprimir los trabajos.

\section{Desarrollo de la innovación}

\subsection{La herramienta Vyew.com}

Vyew.com es una herramienta web que permite reunión en línea, compartir información (imágenes, archivos, documentos,...), y que además permite que los usuarios implicados accedan, contribuyan, los modifiquen, en cualquier momento, bien al mismo tiempo (síncrono) o en momentos distintos (asíncrono). Los usuarios reunidos pueden comunicarse mediante chat, audio y vídeo en función de las necesidades (véase Olmo \& Navarro, 2014). Dados los resultados satisfactorios de dicho trabajo, se ha optado por seguir utilizando esta herramienta para la tutoría virtual. Cabe señalar que la misma ha pasado a ser de pago recientemente para los nuevos usuarios, lo cual no ha sido obstáculo para este trabajo ya que se disponía de cuentas de usuario gratuitas.

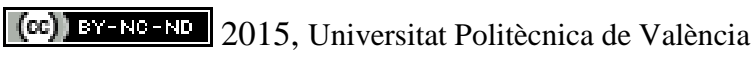

Congreso In-Red (2015) 
Por estos motivos se han probado otras herramientas similares alternativas como las incorporadas en el Google Drive (documentos y dibujos de google, junto con hangouts) o la herramienta de Polireunión de la Universitat Politècnica de València (UPV).

En cuanto a la elección de la herramienta Vyew.com frente a las alternativas sopesadas, observamos que:

- la herramienta de Google Drive carece de audio y vídeo por lo que para comunicarse hay que limitarse al chat o trabajar con el hangouts u otros sistemas de llamada o videoconferencia en paralelo lo que entorpece la tutoría en lenguas extranjeras;

- por otra parte existen aplicaciones online muy adecuadas para comunicación mediante videoconferencia (Firefox Hello) o combinada con el chat (Skype); pero lo que se busca en este trabajo es también poder compartir documentos o escritorio, y trabajar sobre ellos conjuntamente;

- otras aplicaciones permiten compartir escritorio (join.me, screenleap) pero no disponen de comunicación, ni se pueden insertar o compartir documentos;

- la aplicación polireunión de Adobe Connect que ofrece la UPV es muy completa y, si bien permite la grabación de la sesión en vídeo, no permite el acceso a la sala después de terminar la sesión reservada con lo cual no se pueden realizar las tutorías asíncronas;

- existe otra herramienta muy similar y de acceso gratuito (Mashme.tv), que se está probando como alternativa en estos momentos para posteriores trabajos en esta línea.

\subsection{Diseño de actividades}

Se han diseñado dos tipos de actividades para implementar esta aplicación en el desarrollo de las competencias señaladas, enmarcadas en dos asignaturas distintas de la Escuela Técnica Superior de Ingeniería Agronómica y del Medio Natural (ETSIAMN) de la Universitat Politècnica de València, en el curso académico 2014/15. En ambas actividades se ha trabajado en grupos de entre 2 y 4 alumnos. A continuación se describen las mismas, si bien después se ponen en común los análisis y resultados.

\subsubsection{Actividad en REPRESENTACIÓN GRÁFICA EN LA INGENIERÍA (RGI)}

Asignatura troncal de primer curso, en la titulación Graduado en Ingeniería Agroalimentaria y del Medio Rural; 20 alumnos. Grupos de entre 2 y 4 alumnos (a elección).

Tarea: realización, en horario no presencial, de un ejercicio práctico, en lámina de dibujo A4, bien dibujando a mano o con AutoCAD (a elección), consistente en la resolución de un problema práctico de representación y cálculo en el sistema de planos acotados de un embalse de regulación de riego. 
El profesor crea una sala para cada grupo, dando acceso a todos sus miembros. En esta sala, el grupo de alumnos puede trabajar entre ellos mediante reunión virtual, síncrona o asíncrona, durante la realización del trabajo; independientemente de que también puedan reunirse presencialmente.

Y por otra parte, el grupo también debe compartir en la sala la lámina de su ejercicio de dibujo con el profesor (bien escaneada o fotografiada si se trabaja a mano, o bien una captura de pantalla si realiza con AutoCAD); y sobre ella, alumnos y profesor, hacen anotaciones, correcciones (véase la Figura 1), reflexionan y comentan mediante el chat y/o mediante voz. Profesor y alumnos van colaborando de forma asíncrona, y también el grupo puede solicitar tantas tutorías síncronas como necesite durante el trabajo. Se exige que al menos una de ellas sea síncrona, y cuando el ejercicio ya esté terminado.

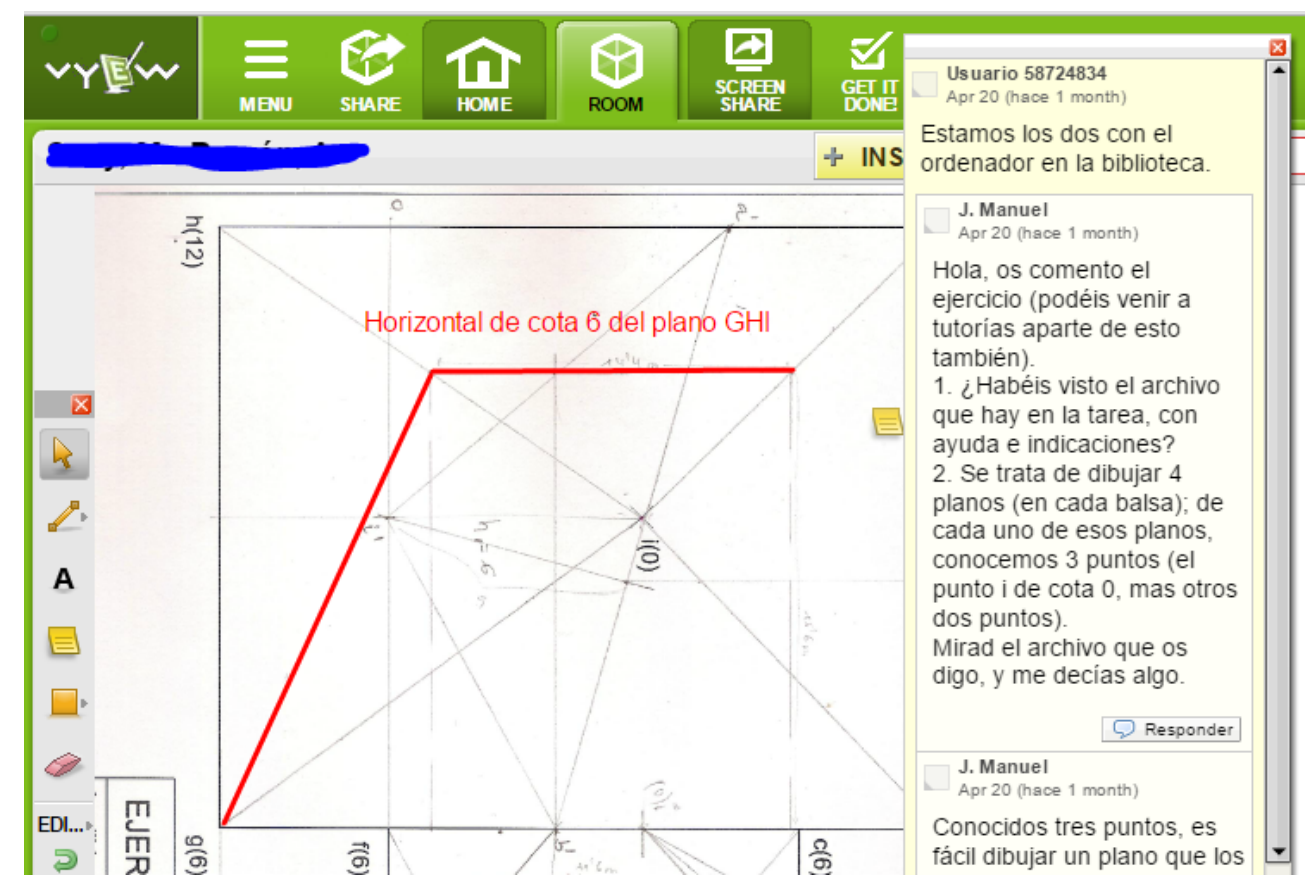

Fig. 1 Aspecto de una página con documento compartido, en una sala de Vyew.com

\subsubsection{Actividad en FRANCÉS II PARA BIOTECNOLOGÍA (FAB)}

Asignatura de 6 créditos, complementos de formación de tercer curso, Graduado en Biotecnología, 14 alumnos. Grupos de dos alumnos.

Tarea: consiste en proponer dentro del sistema de evaluación un trabajo a efectuar por parejas. Al igual que para la actividad anterior, el entorno colaborativo Vyew permite por un

\section{(cc) EY-NC-ND 2015, Universitat Politècnica de València}

Congreso In-Red (2015) 
lado, que los alumnos empiecen a trabajar entre pares y por otro, que lo hagan con el docente para obtener consejos y retroalimentación. El tema planteado para esta asignatura se basa en comparar los planes de estudios de nuestra escuela con uno de una escuela de biotecnología francesa y destacar a nivel de la formación, las ventajas o/y los inconvenientes que conllevaría una estancia en la misma. Para ello, cada grupo debe realizar un trabajo escrito que será proyectado mediante presentación Power Point o similar a la clase y una presentación oral.

Para esta actividad, a la diferencia de la anterior, solo se concierta una tutoría virtual obligatoria por ser la duración de ésta de entre 30 y 90 minutos. Esta tiene lugar unos quince días antes de la exposición oral. En lenguas extranjeras, todas las herramientas comunicativas de la aplicación (el chat y la videoconferencia) se utilizan para corregir los documentos escritos así se practican a la vez la expresión y comprensión oral; la expresión y comprensión escrita y la interacción (véase la Figura 2). Estas características específicas hacen que en el ámbito de los idiomas el tamaño de grupo deba ser reducido. Además de evaluar la tarea, las preguntas realizadas por el docente miden durante el ejercicio el grado de adquisición de las competencias transversales 1 y 6 (véase rúbrica elaborada para tal efecto en el apartado de resultados).

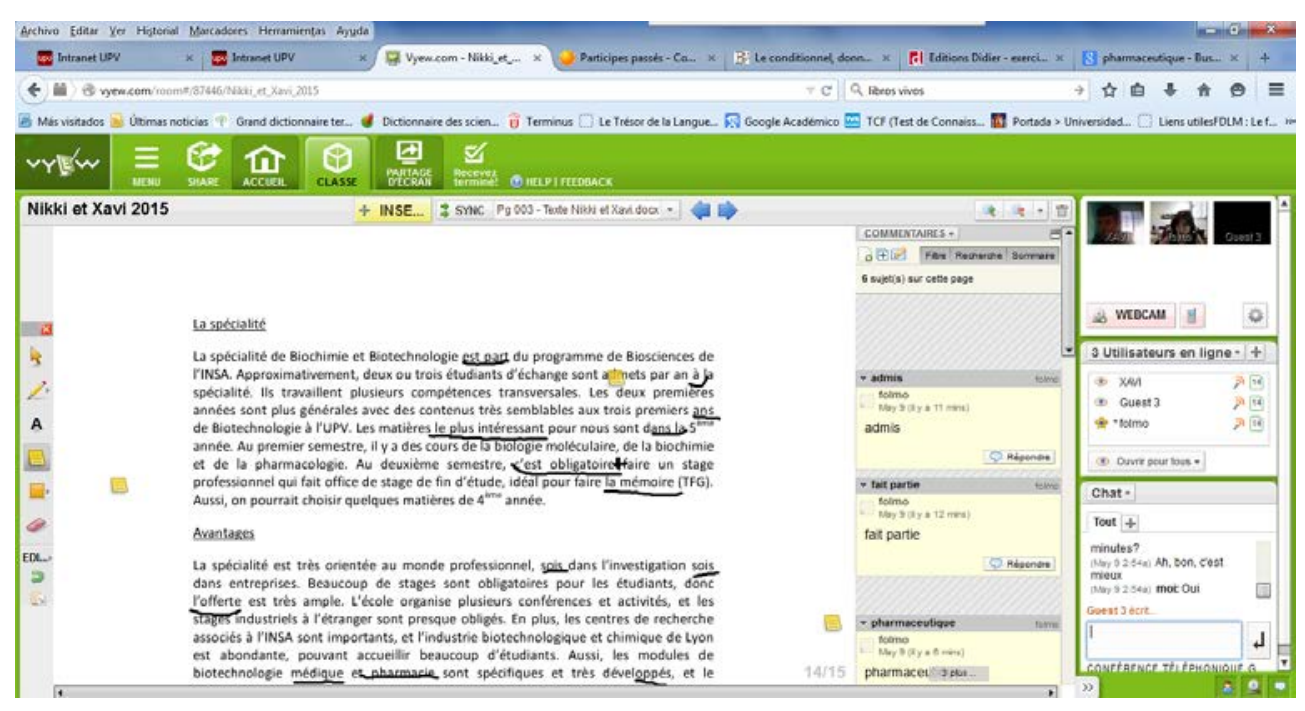

Fig. 2 Ejemplo de tutoría de francés

Después de las actividades propuestas, se utilizaron dos documentos para recoger evidencias acerca de la validez de la herramienta Vyew para la estimación del grado de adquisición de las competencias mencionadas por parte de los estudiantes:

- Una encuesta a los alumnos, a través de PoliformaT, la plataforma educativa de teleformación de la UPV. Ésta consta de siete preguntas de tipo Likert con cinco niveles 
de respuesta (desde totalmente en desacuerdo a totalmente de acuerdo): dos relacionadas con la competencia uno y cinco con la competencia 6.

- Una rúbrica para la evaluación por parte del profesor. Tras haber revisado la literatura correspondiente a la elaboración de las rúbricas, en particular la guía de Palloff i Pratt (2005), proponemos una rúbrica relacionada con la actividad propuesta, es decir, que evalúe las dos competencias descritas. En ambas asignaturas, al final de la tutoría el profesor indaga mediante preguntas orientadas a la cumplimentación de la misma. La rúbrica (véase Tabla 1) se compone de cinco ítems: dos relacionados con la competencia 1 y tres con la competencia 6.

Tabla 1. Rúbrica elaborada para evaluar adquisicion de competencias 1 y 6

\begin{tabular}{|l|l|l|l|l|l|}
\hline & \multicolumn{1}{|c|}{$\mathbf{1}$} & \multicolumn{1}{|c|}{$\mathbf{2}$} & \multicolumn{1}{|c|}{$\mathbf{4}$} & \multicolumn{1}{|c|}{$\mathbf{5}$} \\
\hline $\begin{array}{l}\text { Explica } \\
\text { conceptos } \\
\text { básicos del } \\
\text { tema }\end{array}$ & $\begin{array}{l}\text { No explica } \\
\text { los } \\
\text { conceptos }\end{array}$ & $\begin{array}{l}\text { Explica de } \\
\text { forma } \\
\text { parcial con } \\
\text { un } \\
\text { lenguaje } \\
\text { poco } \\
\text { adecuado }\end{array}$ & $\begin{array}{l}\text { Explica los } \\
\text { conceptos de } \\
\text { una forma } \\
\text { aceptable }\end{array}$ & $\begin{array}{l}\text { Explica los } \\
\text { conceptos de } \\
\text { forma clara, } \\
\text { ordenada }\end{array}$ & $\begin{array}{l}\text { Explica los } \\
\text { conceptos } \\
\text { clara y } \\
\text { ordenadament } \\
\text { e, argumenta } \\
\text { y da opinión }\end{array}$ \\
\hline $\begin{array}{l}\text { Relaciona e } \\
\text { integra los } \\
\text { conocimientos }\end{array}$ & $\begin{array}{l}\text { No integra } \\
\text { ni relaciona } \\
\text { los } \\
\text { conceptos }\end{array}$ & $\begin{array}{l}\text { Integra los } \\
\text { conceptos } \\
\text { con } \\
\text { dificultad }\end{array}$ & $\begin{array}{l}\text { Establece } \\
\text { algunas } \\
\text { conexiones } \\
\text { entre los } \\
\text { conceptos }\end{array}$ & $\begin{array}{l}\text { Es capaz de } \\
\text { relacionar } \\
\text { los } \\
\text { conceptos e } \\
\text { integrarlos } \\
\text { con otros }\end{array}$ & $\begin{array}{l}\text { Es capaz de } \\
\text { relacionar e } \\
\text { integrar los } \\
\text { conceptos } \\
\text { básicos dentro } \\
\text { de esquemas } \\
\text { globales } \\
\text { complejos }\end{array}$ \\
\hline $\begin{array}{l}\text { Realiza las } \\
\text { tareas que le } \\
\text { son asignadas } \\
\text { dentro del } \\
\text { grupo en } \\
\text { plazos } \\
\text { requeridos. }\end{array}$ & $\begin{array}{l}\text { No cumple } \\
\text { las tareas } \\
\text { asignadas }\end{array}$ & $\begin{array}{l}\text { Cumple } \\
\text { parcialmen } \\
\text { te las } \\
\text { tareas } \\
\text { asignadas } \\
\text { o se } \\
\text { retrasa. }\end{array}$ & $\begin{array}{l}\text { Da cuenta en el } \\
\text { plazo } \\
\text { establecido de } \\
\text { los resultados } \\
\text { correspondient } \\
\text { es a la tarea } \\
\text { asignada. }\end{array}$ & $\begin{array}{l}\text { La calidad } \\
\text { de la tarea } \\
\text { asignada } \\
\text { supone una } \\
\text { notable } \\
\text { aportación al } \\
\text { equipo. }\end{array}$ & $\begin{array}{l}\text { Además de } \\
\text { cumplir la } \\
\text { tarea } \\
\text { asignada, su } \\
\text { trabajo orienta } \\
\text { yacilita el } \\
\text { del resto de } \\
\text { miembros del } \\
\text { grupo }\end{array}$ \\
\hline
\end{tabular}




\begin{tabular}{|c|c|c|c|c|c|}
\hline $\begin{array}{l}\text { El grupo } \\
\text { cumple con los } \\
\text { plazos } \\
\text { requeridos }\end{array}$ & $\begin{array}{l}\text { No entrega } \\
\text { el ejercicio } \\
\text { y no realiza } \\
\text { la tarea }\end{array}$ & $\begin{array}{l}\text { No entrega } \\
\text { en plazo y } \\
\text { solicita } \\
\text { ampliación }\end{array}$ & $\begin{array}{l}\text { El ejercicio } \\
\text { está } \\
\text { incompleto una } \\
\text { vez cumplido } \\
\text { el plazo }\end{array}$ & $\begin{array}{l}\text { El ejercicio } \\
\text { está } \\
\text { completo y } \\
\text { en plazo, } \\
\text { pero sin } \\
\text { contrastar su } \\
\text { corrección o } \\
\text { calidad con } \\
\text { el profesor }\end{array}$ & $\begin{array}{l}\text { El ejercicio } \\
\text { está completo } \\
\text { en el plazo y } \\
\text { correcto de } \\
\text { acuerdo a las } \\
\text { indicaciones } \\
\text { del profesor }\end{array}$ \\
\hline $\begin{array}{l}\text { Participa de } \\
\text { forma activa } \\
\text { en los espacios } \\
\text { de encuentro } \\
\text { síncrono del } \\
\text { grupo y con el } \\
\text { profesor, } \\
\text { compartiendo } \\
\text { la información, } \\
\text { conocimientos } \\
\text { y experiencias. }\end{array}$ & $\begin{array}{l}\text { En los } \\
\text { trabajos de } \\
\text { grupo se } \\
\text { ausenta con } \\
\text { facilidad y } \\
\text { su presencia } \\
\text { es } \\
\text { irrelevante }\end{array}$ & $\begin{array}{l}\text { Durante la } \\
\text { sesión, } \\
\text { interviene } \\
\text { poco o } \\
\text { nada, llega } \\
\text { tarde o se } \\
\text { ausenta } \\
\text { momentán } \\
\text { eamente }\end{array}$ & $\begin{array}{l}\text { En general se } \\
\text { muestra activo } \\
\text { y participativo } \\
\text { en los } \\
\text { encuentros del } \\
\text { grupo. }\end{array}$ & $\begin{array}{l}\text { Con sus } \\
\text { intervencion } \\
\text { es fomenta la } \\
\text { participación } \\
\text { y mejora de } \\
\text { la calidad de } \\
\text { los } \\
\text { resultados } \\
\text { del equipo. }\end{array}$ & $\begin{array}{l}\text { Sus } \\
\text { aportaciones } \\
\text { son } \\
\text { fundamentale } \\
\text { s tanto para el } \\
\text { procesos } \\
\text { grupal como } \\
\text { para la } \\
\text { calidad del } \\
\text { resultado. }\end{array}$ \\
\hline
\end{tabular}

\section{Resultados}

En las Figuras 3 y 4 se observan los resultados de las dos primeras cuestiones de la encuesta, referentes a la comprensión e integración, respectivamente, de los conocimientos trabajados durante la actividad. Aparecen ambas asignaturas diferenciadas por colores, así como el resultado global.

En cuanto a la comprensión, el 77 \% de los alumnos opina que la tarea les permitió comprender mejor los aspectos clave. Y respecto a la integración, el 73 \% piensa que le ha ayudado a relacionar e integrar los conocimientos tratados con los que ya tenía. 


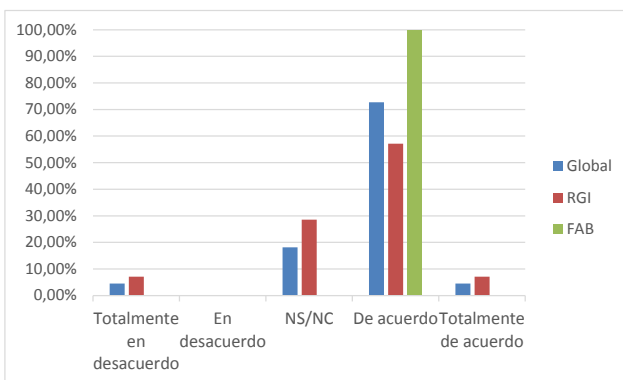

Esta tarea me ha permitido comprender mejor los aspectos clave tratados en el tema

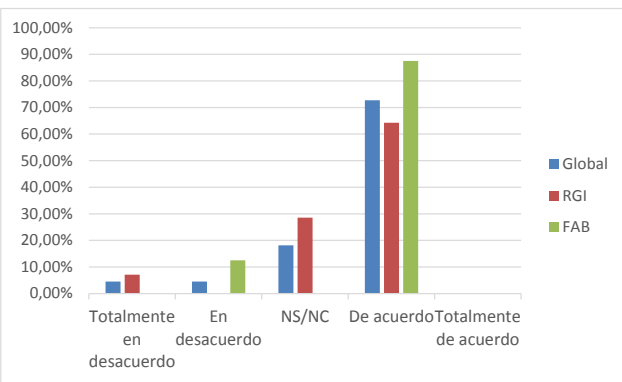

Esta tarea me ha permitido relacionar e integrar los conocimientos que estoy adquiriendo con los anteriores que ya tenía

Fig. 4 Integración

Fig. 3 Comprensión

Respecto a la competencia trabajo en equipo se observan diferencias entre las dos asignaturas. En las preguntas relativas al reparto consensuado de las tareas (Figura 5), el cumplimiento de los plazos establecidos (Figura 6), y el desarrollo del trabajo de forma cooperativa (Figura 7), los resultados en la asignatura Francés para biotecnología (FAB) son más favorables en estos aspectos que en la asignatura Representación gráfica en la ingeniería (RGI). Esto puede ser debido al tipo de actividad propuesto: en el primer caso se trata de un proyecto con varios subapartados, y en el segundo se trata de un ejercicio puntual y más corto, que se presta más a ser resuelto en colaboración directa sin división de tareas.

En ambas asignaturas el alumnado manifiesta estar satisfecho (en un 76 \% en global) con la forma de trabajar en el grupo (Figura 8). 
Desarrollo de competencias transversales mediante tutoría virtual

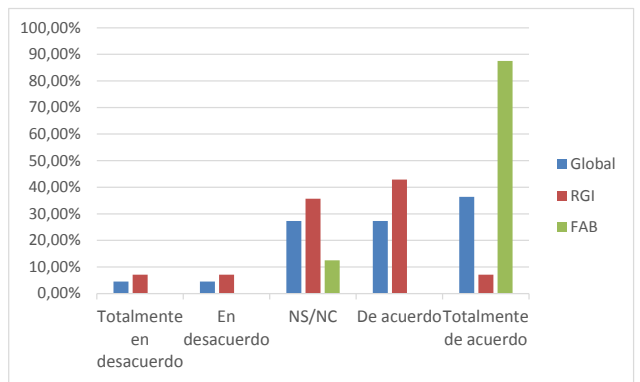

Las subtareas de esta actividad se han repartido de común acuerdo en el grupo

Fig. 5 Reparto de tareas en el grupo

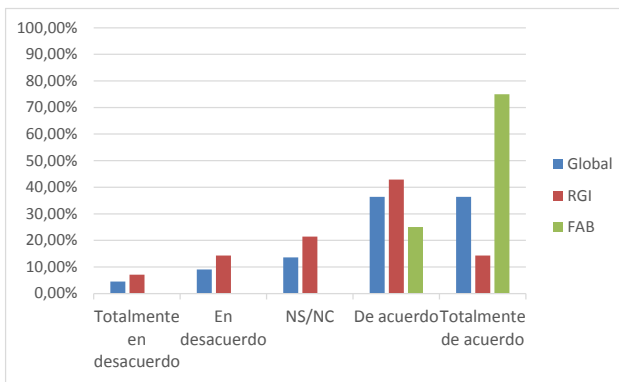

Cada miembro del grupo ha cumplido con la realización de sus tareas en los plazos establecidos

Fig. 6 Cumplimiento de tareas en los plazos establecidos

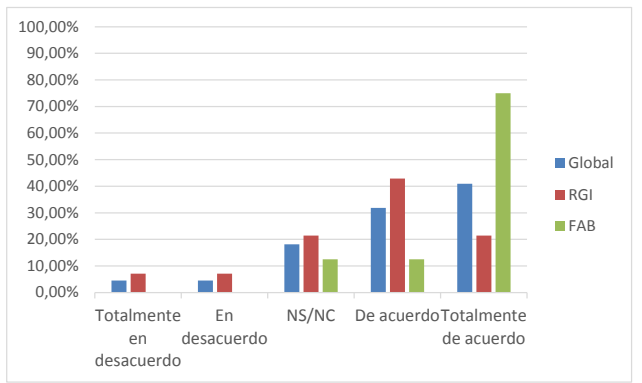

La tarea se ha desarrollado de forma cooperativa

Fig. 7 Trabajo cooperativo

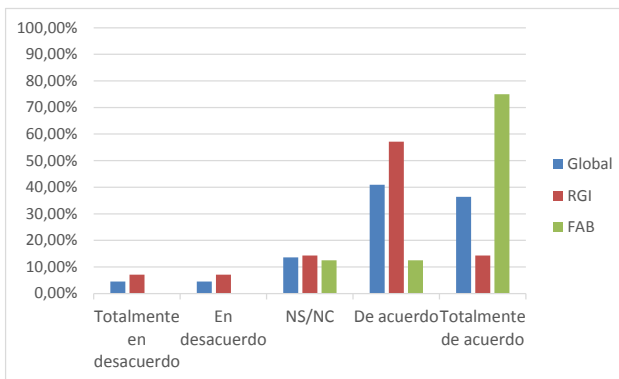

Estoy satisfecho con la forma en que hemos trabajado conjuntamente en el grupo

Fig. 8 Satisfacción con el trabajo conjunto

Por último, el 73 \% está de acuerdo con la afirmación de que el Vyew fomenta el trabajo colaborativo (Figura 9). 


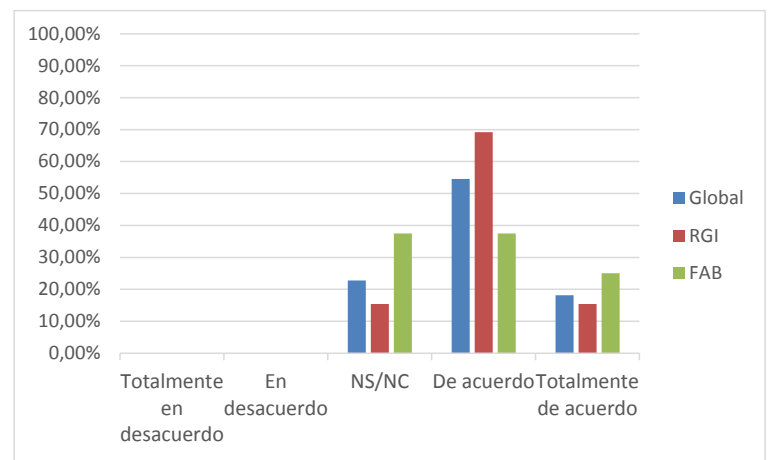

El Vyew.com fomenta el trabajo colaborativo

Fig. 9 Vyew fomenta la colaboración

En cuanto a la rúbrica, los resultados de su aplicación a los trabajos grupales han ido en la misma línea que los de las encuestas en cuanto a la satisfacción con el trabajo en equipo y con la adquisición de la competencia 1. Cabe subrayar que todos los alumnos han tenido una puntuación no inferior a 3 (véase tabla 1) excepto en un par de grupos que no han entregado la tarea y por tanto, no han demostrado haberlas adquirido.

\section{Conclusiones}

Cabe indicar que, aparte de para recoger información acerca de la validez de la herramienta Vyew para la estimación del grado de adquisición de las competencias, las preguntas de la encuesta se han realizado específicamente para que los estudiantes empiecen a tomar conciencia del valor de las competencias transversales en general, y de lo que se espera de ellos en las competencias 1 y 6 en particular.

Los resultados recogidos nos permiten confirmar que la aplicación Vyew ha permitido a los alumnos llevar a cabo las tareas planteadas de forma colaborativa y cooperativa, y con un alto grado de satisfacción con el trabajo realizado. Además, esta forma de comunicación presenta algunas ventajas claras: la falta de necesidad de desplazamiento de los alumnos al centro docente, y en nuestra opinión y por la observación de la experiencia vivida, que en muchos casos los alumnos preguntan con más facilidad las dudas o la explicación de los conceptos más complejos en estas tutorías que en clase presencial o incluso que en las tutorías presenciales.

Por ello se puede decir que este tipo de aplicación es útil para desarrollar la comprensión y la integración. 
También conviene subrayar que las características de esta aplicación permiten ampliar la recogida de evidencias de adquisición de competencias, más allá de la rúbrica o de los resultados de la encuesta, ya que los trabajos presentados (incluyendo los documentos, las conversaciones, las anotaciones y correcciones realizadas) se pueden conservar en las salas utilizadas para cada grupo de trabajo.

Por último, señalar que para trabajos futuros en los que se utilicen herramientas virtuales bien para el desarrollo de competencias transversales o bien con otros fines docentes, se hace necesaria una revisión exhaustiva y continua de herramientas disponibles, puesto que aparecen cada vez con mayor frecuencia y con más novedades, otras cambian a ser de pago, y otras desaparecen.

\section{Referencias}

DiNuCCI, D. (1999). “Fragmented future” en Print. Vol.53 (4), p.32.

GonzÁlez, J. y WagenAAR, R. (eds) (2006). Tuning Educational Structures in Europe II. La contribución de las universidades al Proceso de Bolonia. Bilbao: Universidad de Deusto.

HARRIS, J. y HOFER, M. (2009). “Instructional planning activity types as vehicles for curriculum-based TPACK development” en C. D. Maddux, (ed.). Research highlights in technology and teacher education. Chesapeake, VA: Society for Information Technology inTeacher Education (SITE), pp. 99108.

Olmo, F. y NAVARro, J.M. (2014). “La tutoría virtual en la enseñanza. La individualización de la formación. En Revista del CIDUI, 2.. Girona.

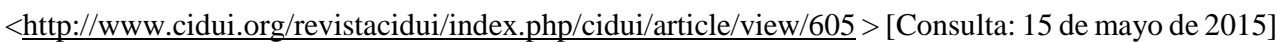

PALLOFF, R. M.; PRATT, K. (2005). Collaborating online. Learning together in community. San Francisco: Jossey-Bass. 